\title{
Vergleiche jenseits des Nationalstaats
}

\section{Methodologischer Kosmopolitismus in der soziologischen Forschung über hochqualifizierte Migration}

\section{Von Anja Weiß ${ }^{1}$}

Zusammenfassung: Als nomothetische Wissenschaft zielt die Soziologie darauf, durch Vergleiche vieler Fälle innerhalb eines übergreifenden Kontextes möglichst breit verallgemeinerbare Erkenntnisse zu erzielen. Dabei werden die Spezifik des Ortes und des Falles in der Regel vernachlässigt. Der Vorteil des soziologischen Zugangs liegt darin, dass auch nicht intendierte und nicht politisierte Effekte sozialer Strukturen sichtbar werden. Problematisch ist, dass Fälle regelmäßig nur im Nationalstaat situiert werden. Das Programm eines methodologischen Kosmopolitismus zielt auf Veränderungen in der Konstruktion von Fällen und Vergleichskontexten und deren Verhältnis zueinander. Auf der Grundlage von Methoden und Befunden aus der ethnographischen und der historisch vergleichenden Forschung schlägt der Artikel vor, Fälle in multiplen Kontexten (u.a. auch transnationalen sozialen Feldern oder postkolonialen Räumen) zu situieren. Vergleiche sollten so angelegt werden, dass empirisch erfasst werden kann, ob und wie der Nationalstaat Fälle strukturiert. Anhand eigener Forschungsprojekte aus der Migrationsforschung werden idealtypische Vergleichsoptionen ,jenseits“ des Nationalstaats diskutiert.

\section{Einleitung}

Eine soziologische Forschung, deren Blickfeld mit dem Rahmen des Nationalstaats identisch ist, bringt systematische Verzerrungen hervor, denn sie kann die Bedeutung des nationalstaatlichen Kontextes für die interessierenden Phänomene nur teilweise untersuchen, da sie seine Grenzen nicht in den Blick nimmt. Bildlich ausgedrückt geht es darum, dass eine Beobachterin, die durch einen Trichter (den am Nationalstaat orientierten Gesellschaftsbegriff) auf eine Ansammlung von Kreisen (die soziale Welt) blickt, Ausschnitte von Kreisen wahrnehmen wird, die oft verwirrend sind. Wenn sie den Trichter ausschließlich so einsetzt, dass die Grenze ihres Blickfelds mit einem oder mehreren besonders wichtigen Kreisen (z.B. nationalen Wohlfahrtsstaaten in der OECD-Welt) übereinstimmt, wird ihr Blickfeld übersichtlicher, aber sie übersieht dann die jeweils ausgeblendeten Kreise und sie kann die Grenze ihres Kreises und die Überschneidungsbereiche und Verbindungen zwischen den Kreisen schlecht erfassen. Der methodologische Nationalismus stellt eine Blickverzerrung dieser Art dar. Er unterstellt, dass sich soziale Phänomene primär durch den Nationalstaat, in dem sie geographisch lokalisiert sind, rahmen lassen.

Dadurch werden systematisch zwei Effekte produziert: Erstens wird die globale Bedingtheit lokaler Phänomene (Robertson 1992; Albrow 1996) leicht übersehen. Zum Beispiel konzentriert sich die soziologische Ungleichheitsforschung auf scheinbar meritokratisch hervorgebrachte Ungleichheiten innerhalb des Nationalstaats und verwendet wenig Augenmerk darauf (Beck 2007; Berger / Weiß 2008), dass die Ungleichheit zwischen Haushalten in der Welt zu etwa $60 \%$ mit dem Staat, in dem sich ein Haushalt befindet, und damit mit ungleicher Geburt

1 Ich danke der Leitung und den Teilnehmenden der Tagungen „Varieties of Reflexive Modernization“ und "Unbounded Methodologies: Methodological Reflections on Research on Overlapping Spaces, Border-Crossing Diffusion and Transnational Histories" (ZiF Bielefeld) sowie den anonymen Gutachtern, Arnd-Michael Nohl, Karin Schittenhelm und Damir Softic für anregende Kritik. Die VWStiftung hat die internationale Studiengruppe „Kulturelles Kapital in der Migration“, deren Ergebnisse hier u.a. präsentiert werden, großzügig unterstützt. 
kovariiert (Milanovic 2008). ${ }^{2}$ Für eine sich als wertfrei verstehende Soziologie wird der methodologische Nationalismus auch deshalb zum Problem, weil diese Art der Blickverkürzung in allzu offensichtlichem Zusammenhang mit postkolonialen Herrschaftsverhältnissen steht, von denen ein großer Teil der soziologischen Zunft profitiert (Bourdieu 1984).

Das „Übersehen“ des Nationalstaates bringt zweitens ein nur scheinbar gegenteiliges Problem hervor: In dem Moment, in dem der Trichterblick nationale Grenzen überschreitet, richtet sich zu viel Aufmerksamkeit darauf, dass zwei oder mehr nationalstaatliche Kontexte in den Blick genommen werden. Dann werden Phänomene als national oder ethnisch spezifisch charakterisiert, die wenig oder nichts mit dem Nationalstaat zu tun haben. Dieses Problem wird in der Ethnologie seit langem reflektiert, ohne dass es als überwunden gelten kann (Wimmer / Glick Schiller 2002; Glick Schiller / Caglar / Guldbrandsen 2006). In der Soziologie wird es in der Migrationsforschung besonders augenfällig. Hier ist es zum Beispiel gang und gäbe, die Bildungsarmut von „Türken“ in Deutschland zu untersuchen und dabei tatsächlich Personen zu meinen, deren Vorfahren sich im Verlauf der letzten 50 Jahre zur Auswanderung aus der Türkei entschlossen haben, die dann nicht nach Großbritannien oder die USA, sondern nach Deutschland gegangen sind, die sich nicht haben einbürgern lassen (können) und die seither nicht zurück- oder weitergewandert sind. Folgt man ihrer Selbstbeschreibung, so würde es sich bisweilen auch um Kommunistinnen oder Kurden handeln. Aus soziologischer Sicht wäre es ebenso gut zu rechtfertigen, die interessierende Gruppe zu den „Armen“, den „Ungebildeten“, den „Ausländern“ oder den „,rassistisch Diskriminierten“ zu zählen. Der Umstand, dass sie nationale Grenzen überschritten haben, lässt aus ihnen „Türken“ werden, so dass auch Phänomene, die sie mit nicht oder anders ethnisch markierten Personen gemeinsam haben, ihrer nationalen Herkunft zugerechnet werden.

Was als problematische Gepflogenheit einer Bindestrich-Soziologie erscheinen mag, wird in der ländervergleichenden soziologischen Forschung grundsätzlicher reflektiert. Wenn sich z.B. empirisch feststellen lässt, dass Japaner länger leben als Amerikaner, sei es Aufgabe der ländervergleichenden Forschung, eine solche Zurechnung auf ,nationale Eigennamen“ durch kausale Erklärungen über analytische Variablen zu ersetzen (vgl. Przeworski / Teune 1970: 10; Goldthorpe 1997 a,b). Bei dem hier angeführten Beispiel lässt sich z.B. zeigen, dass Menschen länger leben, wenn sie mehrfach ungesättigte Fettsäuren konsumieren. Mit Japan oder den USA hat das nur wenig zu tun.

Allerdings kann die ländervergleichende Forschung das Problem besser benennen als lösen. Aufgrund der Verflechtung vieler Variablen im Rahmen einer längerfristigen und pfadabhängigen historischen Entwicklung bei gleichzeitig niedriger Fallzahl lassen sich auch im mittlerweile sehr gut entwickelten Paradigma der Mehrebenenanalyse nationale Eigennamen nicht vollständig durch aufgeklärte Kausalbeziehungen zwischen analytischen Variablen ersetzen. Daher sieht sich auch die international vergleichende soziologische Forschung genötigt, das interessierende inhaltliche Phänomen, z.B. den „Lebenslauf“, durch einen nationalen Eigennamen , in Deutschland“ zu ergänzen. Der nationale Eigenname steht dann einerseits für den deutschen Wohlfahrtsstaat - also die gewünschte analytische „Variable“ - und andererseits für den häufig recht großen Rest an historisch gewordener Besonderheit, der sich im Rahmen variablenlogischer Vergleiche nicht aufklären lässt (vgl. Goldthorpe 1997 a: 17).

Die ländervergleichende Forschung reflektiert auch das erste hier angesprochene Problem, die globale Bedingtheit scheinbar lokaler Phänomene, als „Galton-Problem“ (Ebbinghaus

2 Damit ist allerdings nicht gesagt, dass die Zugehörigkeit zu einem Staat Ungleichheit zu 60 \% erklärt. Das Problem besteht gerade darin, dass sich die Ursachen für diesen Anteil an weltweiter Varianz nicht klären lassen, solange sich die globale Ungleichheitsforschung auf Vergleiche zwischen Nationalstaaten beschränkt. 
1996; Goldthorpe 1997 b). Die Festlegung auf Länder als Einheiten des Vergleichs erschwert es aber grundsätzlich, grenzüberschreitenden Verflechtungen gerecht zu werden.

Die hier angerissene Kritik mag genügen, um in das mittlerweile breit erkannte Problem des methodologischen Nationalismus einzuführen. Die größere Schwierigkeit liegt jedoch darin, das Problem zu überwinden. Dabei steht eine soziologische empirische Forschung, die ihre Erkenntnisse als potentiell allgemein gültig denkt und die ihren Gegenstand in sehr großen und unbestimmten Kontexten verortet, vor größeren Problemen als ideographische Disziplinen wie die Ethnologie, die Geschichte und Teile der Politikwissenschaft, die von vorneherein mehr Aufmerksamkeit auf die Spezifik von Kontexten verwenden oder diese selbst zu ihrem Gegenstand machen (Wallerstein 2004). Dieser Artikel setzt sich in grundlagentheoretischer Absicht mit der Frage auseinander, wie eine soziologische empirische Forschung beschaffen sein muss und kann, die die Verkürzungen des methodologischen Nationalismus überwindet und die damit auf die interdisziplinären Herausforderungen der Transnationalismusforschung und des methodologischen Kosmopolitismus antwortet.

Im nächsten Teil des Beitrags wird am Beispiel der Lebensverlaufsforschung präzisiert, wie die soziologische Forschung Fallvergleiche in Kontexte einbettet. Das Programm eines methodologischen Kosmopolitismus zielt auf Veränderungen in der Konstruktion von Fällen und Vergleichskontexten und deren Verhältnis zueinander, die im dritten Teil expliziert werden. Hier kann an Methoden und Befunde aus der ethnographischen und der historisch vergleichenden Forschung angeschlossen werden. Um die Vorschläge für soziologisch vergleichende Forschungsdesigns nicht im Abstrakten zu belassen, werden im vierten Teil idealtypische Vergleichsoptionen ,jenseits“ des Nationalstaats anhand eigener Forschungsprojekte vorgestellt. Diese Beispiele haben ebenso wie der hier gewählte Bezugsbereich der Lebensverlaufsund Individualisierungsforschung exemplarischen Charakter, d.h. sie dienen dazu, die hier behandelten methodologischen Probleme zu illustrieren, können aber dem Gegenstandsbereich inhaltlich nicht gerecht werden. Am Schluss wird das methodologische Anliegen des Artikels bekräftigt: Das methodologische Repertoire der Soziologie sollte so weiter entwickelt werden, dass die Anforderungen einer transnational und ethnographisch orientierten Soziologie und ihrer Nachbardisziplinen auch im Kern soziologischer Forschung umgesetzt werden können.

\section{Die implizite und explizite Kontextualisierung von Vergleichen in der Soziologie}

Jegliche beobachtende Wissenschaft steht vor der Frage, wie sie aus der unendlichen Fülle von Sachverhalten solche abgrenzt, die sie als „Fall“ ansieht, und welche Fälle sie hinsichtlich welcher Kriterien miteinander vergleicht. Ein Teil dieser Fragen wird in der methodologischen Literatur ausführlich diskutiert (Mill 1973 [1843]; Matthes 1992; Nohl 2007). Zum Beispiel wird erörtert, was ein Fall ist, welche Fälle als maximale oder minimale Kontraste verglichen werden sollten, und ob das gewählte Vergleichsmoment sinnvoll ist. Man behandelt also die Frage, ob es sinnvoll ist, Äpfel und Birnen zu vergleichen und welche Vergleichsmomente beiden Früchten gerecht werden. Systematische Vergleiche sind für die soziologische Forschung besonders bedeutsam, da die Soziologie so einen Anspruch auf statistische Repräsentativität oder auf inhaltliche Generalisierbarkeit (Przyborski / Wohlrab-Sahr 2008: 311ff) begründet. Als nomothetische Wissenschaft ist die soziologische Forschung weniger an den Besonderheiten spezifischer Fälle interessiert als an fallübergreifend verallgemeinerbaren Erkenntnissen.

Die Frage nach geeigneten Vergleichsmomenten beinhaltet Entscheidungen darüber, in welchem sozialen Kontext und in welchem geographischen Rahmen soziologische Vergleiche überhaupt sinnvoll sind. Diese Frage hat in der soziologischen methodologischen Diskussion wenig Aufmerksamkeit auf sich gezogen (Scheuch 1968; Pries 2008 b). In der Forschungs- 
praxis sind implizite Rahmungen am häufigsten, da unmittelbar einzusehen ist, dass man unter pragmatischen Gesichtspunkten nicht alles mit allem vergleichen kann. Niemand wird alle Äpfel und Birnen untersuchen, sondern man nimmt die leicht erreichbaren und nächstliegenden. Genauso ist es bei sozialen Sachverhalten. Wer sich für Lebensläufe in der Moderne interessiert, wird nicht Lebensläufe in der Welt untersuchen, sondern vermutlich Lebensläufe in München oder allenfalls in Deutschland. Auch pragmatisch begründete Beschränkungen des geographischen und zeitlichen Referenzrahmens gehen aber Hand in Hand mit konzeptionellen Verengungen. Eine sozialwissenschaftliche Forschung, die Lebensläufe, ,in Deutschland" untersucht, muss sich nur am Rande mit der Frage auseinandersetzen, ob und wie sich die Entwicklung von Menschen überhaupt weltweit als individualisierter Lebenslauf erfassen lässt. Und auch in der Forschungspraxis wird sie selten auf Personen stoßen, die nicht bereit oder fähig sind, ihre „Biographie“ (Bourdieu 1991) zu schildern. In den meisten Fällen nimmt der methodologische Nationalismus der Soziologie genau diese Form an: Pragmatisch und konzeptionell ist es am einfachsten, im Rahmen des Nationalstaats zu forschen. Da der nationale Rahmen in der Alltagspraxis institutionalisiert ist und da auch die Sozialwissenschaft überwiegend in wenigen starken Nationalstaaten stattfindet, die ihre Ergebnisse dann als ,,allgemein gültig" exportieren, funktionieren methodologisch nationalistische Forschungsdesigns in der soziologischen Forschung reibungslos.

Grundsätzlich ist auch wenig gegen eine geographische und konzeptionelle Selbstbeschränkung von Vergleichen einzuwenden. Wenn man den Übergang von Jugendlichen zwischen Schule und Beruf für den Bezirk Unterfranken untersucht, handelt es sich um eine Frage von lokalem Interesse und es ist unwahrscheinlich, dass die Ergebnisse einer solchen Studie auf „Lebensläufe als solche“ oder „Lebensläufe in der Moderne“ verallgemeinert werden. Hier sollen nicht solche pragmatischen Blickverengungen kritisiert werden, sondern der Umstand, dass die gesellschafts- und grundlagentheoretisch orientierte Sozialwissenschaft nicht selten ähnlich verfährt. So untersuchte der Bremer SFB zur Lebenslaufforschung Lebensläufe in Deutschland und wenigen OECD-Ländern ohne Aufmerksamkeit für migrierende Bevölkerungsgruppen (Leisering / Walker 1998; Heinz 2000), die auch nach den damaligen Statistiken schon ein Zehntel der jungen Generation ausmachten. Die neuere Forschung zum Lebensverlauf führt Ländervergleiche - erneut ohne eine Auseinandersetzung mit Migration - durch, die sich auf die OECD-Welt beschränken (Blossfeld et al. 2008). ${ }^{3}$

Das Beispiel der ertragreichen und anspruchsvollen Forschung zum Lebensverlauf wird hier nicht deshalb angeführt, weil es besonders negativ wäre, sondern weil es typisch für die Selbstverortung einer grundlagentheoretisch orientierten soziologischen Forschung ist: In der Theoriebildung der Lebensverlaufsforschung wird die Bedeutung einer entwickelten Wohlfahrtsstaatlichkeit für die Herausbildung von Lebensläufen durchaus diskutiert (Kohli 1985; Mayer / Müller 1986; Kohli 1994). Die empirische Forschung beschränkt sich dann aber auf Lebensverläufe im Rahmen spezifischer nationalstaatlicher Institutionensysteme, und Lebensverläufe, die Ländergrenzen überschreiten, werden kaum untersucht. Eine Reflexion dazu, inwiefern der universelle Anspruch ihrer Erkenntnisse angesichts einer auf die OECD-Welt beschränkten Forschung relativiert werden müsste, unterbleibt. Die resultierende Ambivalenz reicht bis in konkrete Formulierungen hinein: Der Korpus von Publikationen macht deutlich, dass sie sich auf ,entwickelte Industriegesellschaften“ (Blossfeld et al. 2008: 667) beschränken. Zugleich suggerieren geläufige Titel Globalität - so das „Globalife“- Projekt von Blossfeld et al. - oder auch eine Gültigkeit für die Moderne schlechthin: „The dynamics of modern society" (Leisering / Walker 1998).

Die eine Seite des methodologischen Nationalismus, das „Übersehen“ impliziter Selbstbeschränkungen auf den (Vergleichs-)Rahmen entwickelter Nationalstaaten, bildet sich direkt in

3 Die Lebensverläufe von Migranten werden in gesonderten Studien untersucht (Verwiebe 2004). 
empirischen Forschungsdesigns ab, die sich forschungspragmatisch auf einen Ausschnitt der Welt beschränken, dann aber in der Tradition nomothetischer Wissenschaftlichkeit (zur Kritik vgl. Wallerstein 2004) Theorien für ,die“ soziale Welt insgesamt entwickeln. Wie sieht es mit der anderen Seite aus: der übermäßigen Zurechnung empirischer Phänomene auf den Nationalstaat? Auch dieses Problem lässt sich an konkreten Forschungsfragen präzisieren. Eine Stärke der Lebensverlaufsforschung ist der systematische Vergleich nationaler Wohlfahrtsstaaten und Bildungssysteme im Paradigma der Mehrebenenanalyse. Wer z.B. die Lebensverläufe von Hartz IV-Empfängern untersucht, ist an den Auswirkungen eines bestimmten sozialstaatlichen Instruments auf den Lebensverlauf interessiert. Dieses Instrument existiert so nur in Deutschland und kann auch nur da untersucht werden. In diesem Fall ist es sinnvoll, den empirischen Gegenstand der Forschung explizit nur in einem bestimmten nationalstaatlichen Rahmen zu untersuchen. Außerdem sind Mehrebenenanalysen hilfreich, die explizit ländervergleichend verfahren, denn auch die sozialpolitischen Instrumente anderer Wohlfahrtsstaaten stehen in engem inhaltlichem Zusammenhang mit dem nationalstaatlichen Rahmen und der Ländervergleich eröffnet die Chance, die nationalstaatliche Spezifik sozialpolitischer Instrumente herauszuarbeiten. Erlinghagen und Knuth (2010: 72) können z.B. zeigen, dass der Anteil der Personen im erwerbsfähigen Alter, die von staatlichen Transferzahlungen leben, im Ländervergleich erstaunlich stabil ist, auch wenn die Begründungen und Instrumente der Transfers stark variieren. Ein solcher Ländervergleich akzentuiert die Besonderheit spezifisch deutscher sozialpolitischer Instrumente und er trägt zu verallgemeinerbaren Erkenntnissen über die Handlungsspielräume von Sozialpolitik in Wohlfahrtsstaaten bei.

Schwierig wird es dann, wenn Phänomene im Paradigma einer explizit ländervergleichenden Mehrebenenanalyse untersucht werden, die teilweise, aber nicht eindeutig und ausschließlich durch nationalstaatliche Kontexte geprägt sind (Hopkins / Wallerstein 1967: 54). Die Individualisierung des Lebenslaufs ist ein solches Phänomen, denn mit Individualisierung ist ein Prozess bezeichnet, in dessen Verlauf Menschen insofern zu Individuen werden, als sie in ein direktes und weniger stark durch intermediäre Gruppen und Institutionen vermitteltes Verhältnis zu Staatlichkeit treten (Beck 1986; Wohlrab-Sahr 1997; Beck 2007). Dieser Prozess wird nicht allein durch Staaten angestoßen, aber er ist durch die Besonderheiten einzelner Staaten geprägt. Weil Individualisierung nicht eindeutig auf spezifische Staaten zugerechnet werden kann, kann man Individualisierungsprozesse nicht wie wohlfahrtsstaatliche Sicherungssysteme oder nationale Bildungssysteme behandeln. Während man also Hartz IV sinnvoll und mit Recht als deutsches sozialpolitisches Instrument kennzeichnen und es mit amerikanischen ,workfare“ Programmen vergleichen kann, wäre es wenig überzeugend, von deutscher, niederländischer, britischer und japanischer Individualisierung zu sprechen. Die am Beispiel der Lebenslaufforschung dargestellte Praxis, konzeptionell von der Welt zu sprechen, und dann empirisch OECD-Länder zu vergleichen, tut aber genau das. Sie konzentriert sich nicht auf Phänomene, die sich wie sozialpolitische Instrumente eindeutig auf einen nationalen Referenzrahmen beziehen lassen, sondern sie untersucht umfassende soziologische Fragestellungen wie den Lebenslauf. Auch hier kann man probehalber unterstellen, dass Lebensläufe „in“ Deutschland, ,in“Frankreich oder ,in“Großbritannien spezifischen Mustern folgen, die durch die jeweiligen wohlfahrtsstaatlichen Systeme strukturiert werden. Genauso plausibel ist es aber, Lebensläufe in transnationalen Feldern zu situieren oder in einer durch die BolognaBeschlüsse geprägten europäischen Bildungslandschaft (Beck / Grande 2004: 163ff) oder in global expandierenden amerikanischen Bildungsinstitutionen (Mau 2007) oder in der Reflexiven Moderne (Bonß et al. 2004).

Die soziologische Forschung untersucht nur selten Phänomene, die wie die Übergangsquoten in Unterfranken ausschließlich lokale Bedeutung haben, oder die wie Hartz IV eindeutig auf einen wohlfahrtsstaatlichen institutionellen Rahmen bezogen werden können. Sehr viel häufiger werden Phänomene wie der Lebenslauf betrachtet, die durch den Nationalstaat und 
das Staatensystem mit strukturiert werden, die aber nicht eindeutig und ausschließlich in einem bestimmten Nationalstaat verortet werden können. Das wirft die Frage auf, wie soziologische Vergleiche in Kontexte eingebettet sein können und sollen.

\section{Das Programm eines methodologischen Kosmopolitismus und seine Umsetzung in der soziologischen Forschung}

Das Verhältnis zwischen Fällen und den Kontexten, in denen sie zum Zwecke des Vergleichs eingebettet werden, nimmt in der soziologischen Forschung spezifische Formen an. Als nomothetische Wissenschaft zielt die Soziologie darauf, durch systematisches Vergleichen möglichst breit verallgemeinerbare Erkenntnisse zu erzielen. Ein Teil der soziologischen Forschung unterstellt implizit, dass regionale oder nationalstaatliche Rahmen Vergleichbarkeit gewährleisten, neigt dann aber doch dazu, eine größere und teils sogar globale Gültigkeit ihrer Ergebnisse zu suggerieren. Ein anderer Teil der soziologischen Forschung folgt dem Paradigma der international vergleichenden Mehrebenenanalyse und nimmt Kontexte explizit in den Blick. Allerdings kommen hier nur Nationalstaaten der OECD-Welt, ihre Untergliederungen und Zusammenfassungen als Vergleichsrahmen in Frage. Wenn das untersuchte Phänomen in engem Zusammenhang mit national segmentierten Institutionensystemen steht, ist das unproblematisch. Bei der Vielzahl soziologischer Gegenstände, die teilweise, aber nicht primär und ausschließlich durch den Nationalstaat gerahmt werden, hat die Gepflogenheit der impliziten oder expliziten Rahmung durch den Nationalstaat jedoch mit Recht Kritik auf sich gezogen. U.a. kann die Frage, ob und wie Staatlichkeit ,jenseits“ spezifischer Nationalstaaten diese Gegenstände strukturiert, schlecht geklärt werden, wenn sie ausschließlich im Rahmen von Nationalstaaten verortet werden.

Beck und Grande (im gleichen Band) entwerfen ein interdisziplinäres sozialwissenschaftliches Programm, in dem „Fälle“ als Prozesse oder Strukturen gefasst werden, die in heterogenen Kontexten situiert werden müssen. Der Nationalstaat kann als rahmender Kontext in Frage kommen, muss dann aber in seinem Verhältnis zu anderen rahmenden Kontexten reflektiert werden („embedding the national“). Auch kommt nicht allein der Nationalstaat als Kontext für soziologische Forschung in Betracht (,replacing the national“). Von der bisherigen in der soziologischen Forschung überwiegenden Praxis unterscheidet sich dieses Szenario in drei Hinsichten: Erstens wird der Begriff des Falles auf Gegenstände ausgeweitet, deren ,Einheitlichkeit" schwer zu benennen ist. Prozesse wie Individualisierung oder Strukturen wie transnationale soziale Netzwerke sind weniger eindeutig voneinander abzugrenzen als z.B. Personen, Lebensläufe oder selbst Familien. Zweitens werden die Kontexte, in denen Fälle situiert sind, weder allein als Nationalstaaten noch als abstrakte Weltgesellschaft gedacht, sondern z.B. auch als transkontinentale (Gilroy 1993) oder transnationale soziale Räume (Pries 2008 b). Drittens wird das Verhältnis zwischen Fällen und Kontexten mehrdeutig, d.h. Fälle können und sollten in mehr als einem Kontext situiert werden.

Dieses methodologische Programm knüpft an empirische und methodologische Entwicklungen in der ethnographischen und der makrosozial und historisch vergleichenden Forschung an. Die Ethnographie findet ihren Gegenstand an einem „Ort“, in den sie durch vertiefende Feldforschung eintaucht. Angesichts der Herausforderung dieser Methode durch Globalisierungsprozesse wird in der Ethnographie diskutiert, wie man Fällen gerecht werden kann, die sich nicht an einem territorialen Ort lokalisieren lassen, sondern die sich über verschiedene Orte hinweg erstrecken. Im Vorschlag der multi-sited ethnography mündete das zunächst in die berühmten followings: follow the people, the thing, the metaphor, the story, the biography, the conflict (Marcus 1995: 106ff).

So sollen u.a. auch makrosoziale Strukturen erkennbar werden, die sich nicht unmittelbar im Fall dokumentieren. Eine systematische Erfassung translokaler und/oder makrosozialer 
Strukturen wird jedoch durch die Zufälligkeit der Followings erschwert: „What ties together fieldwork locations is the ethnographer's discovery of traces and clues, her logic of association" (Gille / Ó Riain 2002: 286). Solange die Forschung v.a. expliziten und durch Reflexion erkennbaren Verbindungen folgt, kann sie der historischen Gewordenheit und der nicht-lokalen und / oder makrosozialen Strukturiertheit des Falles kaum gerecht werden. Neuere Arbeiten zur ,global ethnography“(Gille / Ó Riain 2002) plädieren daher für einen multi-sited approach, der soziale Beziehungen verschiedener räumlicher Reichweite und inhaltlichen Abstraktionsgrades berücksichtigt. Dafür müssen u.a. politische Prozesse des ,,place making“ einschließlich der (elitären) Akteure dieses ,place making“ und historisches Material in die ethnographische Forschung einbezogen werden.

Wie eine solche Forschung aussehen könnte, wird an Gilles eigenen Studien, aber auch in Teilen der transnationalen Migrationsforschung erkennbar. Glick Schiller, Caglar und Guldbrandsen (2006) schlagen z.B. vor, kleine Städte als Einstiegspunkte in transnationale soziale Felder zu nutzen. Sie reflektieren die geographische Einbettung ihres Falles, indem sie die Position der untersuchten Kleinstädte in regionalen und globalen Standortkonkurrenzen analysieren. Wegen dieser Konkurrenzen bieten kleine Städte den in ihnen sichtbar lebenden Migranten andere Opportunitätsstrukturen als global cities. Außerdem stellen Glick Schiller et al. theoretisch von der „ethnic lense“ auf eine offenere Rahmung ihres Falles um. Die von ihnen untersuchten fundamentalistischen christlichen Gemeinden bestehen zwar großenteils aus Migranten, werden aber nicht als ethnische Gemeinschaften, sondern nur als universalistisch orientierte Netzwerke wiedergeborener Christen verständlich.

Eine ethnographische Studie von Lebensläufen würde einen Ausgangspunkt wie München wählen, um durch Feldforschung kulturelle Konventionen, Identitäten und institutionelle Strukturen zu erfassen, die an diesem Ort zusammenwirken, um Lebensläufe zu strukturieren. Im Unterschied zur soziologischen Lebensverlaufsforschung würde eine Ethnographie schnell darauf stoßen, dass etliche Münchener aus Familien mit Migrationshintergrund stammen und könnte dann ihren Biographien über Grenzen hinweg folgen und die Bedeutung des Ausländerrechts für deren Lebensläufe thematisieren. Eine ,global ethnography“ würde darüber hinaus theoriegeleitet nach der historischen und überregionalen Bedingtheit der Münchener Lebensverläufe fahnden. Allerdings kämpft die ethnographische Tradition mit der Tendenz, sich auf Zusammenhänge und Identifikationen, die von den Handelnden selbst angesprochen werden, zu konzentrieren.

Der größere Teil der soziologischen Forschung bildet hierzu das genaue Gegenteil. Sie erschließt Kontextbezüge nicht für einen sozial-räumlich eingebetteten Fall, sondern sie spaltet die ethnographische Falldefinition „Lebensläufe in München“ in die Komponenten Fall und Kontext auf: Die Lebensläufe werden zu dekontextualisierten Fällen, die in großer Zahl in einem möglichst weiträumigen und abstrakten Kontext platziert werden. Aus dem Vergleich vieler oder systematisch variierender Fälle will die Soziologie auf sie strukturierende kausale Mechanismen schließen. Dabei wird die Spezifik des Ortes und des Falles in der Regel vernachlässigt. Es besteht aber die Chance, nicht intendierte und nicht politisierte Nebenfolgen sozialer Strukturen auf Lebensverläufe zu explizieren.

Damit verfügen Ethnographie und soziologische Forschung über sich potenziell ergänzende Zugänge zur sozialen Welt. Ethnographische Studien nutzen in der Regel Daten, die dem Paradigma soziologischer Forschung entstammen, und die ethnographische Methode gewinnt in der Soziologie immer mehr Anhänger. Das Erkenntnisinteresse dieses Artikels geht jedoch über eine Arbeitsteilung zwischen Disziplinen und Methoden hinaus. Vielmehr soll der soziologische Zugang zur sozialen Welt so weiterentwickelt werden, dass auch (systematische) soziologische Vergleiche (vieler) Fälle dem Vorwurf des methodologischen Nationalismus entkommen können. 
Die Diskussion über eine global ethnography macht deutlich, dass Fälle durch eine Mehrzahl variierender Vergleichskontexte auch jenseits des Nationalstaats strukturiert sind. Diese Erkenntnis ließe sich durchaus auch in soziologische Vergleiche übersetzen. Hierfür müssen Fälle unter anderem auch in Kontexten jenseits des Nationalstaats platziert werden. Die historisch und makrosozial vergleichende Forschung hat mittlerweile zahlreiche Kontexte identifiziert, die sich nicht mit dem Nationalstaat zur Deckung bringen lassen. Sie werden hier nach dem Ausmaß gegliedert dargestellt, in dem sie sich konzeptionell an den Nationalstaat und den eng mit ihm verbundenen Begriff der Gesellschaft anlehnen. Analog zum Nationalstaat (a) kann man an Makro-Regionen und supranationale Einheiten wie die EU denken. Diese Art von makrosozialen Kontexten ist Analysen im Paradigma der Mehrebenenanalyse zugänglich. Hier werden Fälle lediglich neben dem Nationalstaat weiteren makrosozialen Kontexten zugeordnet.

Die politikwissenschaftliche Forschung zum Wandel von Staatlichkeit (Zürn / Leibfried 2006) zeigt, dass territorial begrenzte nationalstaatliche Container nur eine Form von Staatlichkeit sind. Politische Steuerung (,governance“) (Botzem et al. 2009) und an diese sich anlehnende gesellschaftliche Öffentlichkeiten und Akteursnetzwerke können auch als transnationale und uneindeutig abgegrenzte Kontexte (b) begriffen werden. Zum Beispiel muss man die EU nicht als Ansammlung von Nationalstaaten verstehen, sondern kann sie auch als Institution begreifen, die auf sich kontinuierlich verschiebende Grenzzonen ausstrahlt (Beck / Grande 2004). Auch die historisch vergleichende Analyse von Zivilisationen (Eisenstadt 2000; Schwinn 2006) kann Zivilisationen als uneindeutig abgegrenzte kulturelle Räume denken, die nicht vorschnell mit Nationalstaaten identifiziert werden müssen. In diesem Zusammenhang können dann z.B. der Katholizismus, der so genannte ,islamische Kulturkreis“, aber auch der Sozialismus Fälle rahmen. Da der Rahmen dieser Kontexte unscharf ist, geraten quantitativ empirische Mehrebenenanalysen hier an die Grenzen ihrer Möglichkeiten. ${ }^{4}$

Wenn man die postkoloniale Kritik ernst nimmt, werden makrosoziale Kontexte nicht nur in ihren Grenzen diffus, sondern sie sind auch auf mehr als ein territoriales Zentrum bezogen. Postkoloniale Räume und Staaten (c) (vgl. den Beitrag von Römhild in diesem Band) zeichnen sich dadurch aus, dass sie durch die Kolonialgeschichte und durch fortbestehende asymmetrische Verflechtungen zwischen ehemaligen Kolonien und Kolonisatoren geprägt bleiben. Die von Randeria (2003) so bezeichneten „Cunning States“ entwickeln ihre Politik z.B. sowohl im Verhältnis zu „Geberländern“ als auch im Verhältnis zur einheimischen Wahlbevölkerung. Zonen schwacher Staatlichkeit (Beisheim / Schuppert 2007) sollten grundsätzlich als postkoloniale Räume und nicht - wie in der ländervergleichenden soziologischen und ökonomischen Forschung ${ }^{5}$ überwiegend praktiziert - als Nationalstaaten begriffen werden. Dort haben Regierungen nur geringe Handlungsspielräume und die „Gesellschaft“ ist durch War-Lords, Geberländer und Nicht-Regierungsorganisationen - also durch lokale und globale Gegebenheiten zugleich - geprägt. Das Argument betrifft aber auch die Staaten des Zentrums, die sich als autonome Einheiten definieren, obwohl sie durch (post-)koloniale Verflechtungen geprägt bleiben. Zum Beispiel gerät der Umstand, dass Europa soziale Konflikte durch eine Abwanderung benachteiligter Bevölkerungsgruppen in die Kolonien externalisieren konnte, erst in den Blick, wenn man auch das Zentrum in postkolonialen Vergleichskontexten situiert (Boatcă 2009).

4 Das Paradigma quantitativ empirischer Mehrebenenanalysen setzt eindeutige Grenzziehungen voraus, weil die relevanten empirischen Maßzahlen in der Regel als Durchschnittswerte erhoben und prozessiert werden. Andere methodische Zugänge sind aber durchaus in der Lage „big structures“ and „larges processes“ in „huge comparisons“ (Zitate aus dem Titel von Tilly 1984) zu vergleichen (Ragin 1987).

5 Die gängige Praxis, im Rahmen von Ländervergleichen Mittelwerte auch für schwache Staaten abzubilden (United Nations Development Programme 2007), erfüllt alle Kriterien eines methodologischen Nationalismus. 
Schließlich wäre zu prüfen, ob Fälle auch in schwer lokalisierbaren Kontexten ohne ausgeprägte Staatlichkeit (d) platziert werden können. Diese Überlegung betrifft die Frage, unter welchen Umständen transnationale soziale Räume (Pries 2008 a), ,circuits“ (Faist 2000) und Felder (Levitt / Glick Schiller 2004) Vergleiche rahmen können (Pries 2008 b). Auch Urrys Plädoyer für eine Soziologie, die Mobilitäten statt Strukturen untersucht (Urry 2000) und Albrows Ausführungen zu „socioscapes“(Albrow 1997) wären daraufhin zu prüfen, ob und wie sich Fälle in solchen Kontexten situieren lassen. Außerdem kommen globalisierte Professionen (Iredale 2001) als Kontexte in Betracht, wenn man unterstellt, dass sie in eine transnationale Institutionenbildung eingebettet sind (Teubner 1997; Quack 2007).

Empirische Arbeiten zu makrosozialen Kontexten jenseits des Nationalstaats setzen sich intensiv mit der Besonderheit dieser Kontexte auseinander und sie untersuchen, ob und wie diese Kontexte den Nationalstaat eher ergänzen oder ersetzen. Sie machen also den Kontext selbst zum sie interessierenden Fall und verwenden relativ wenig Aufmerksamkeit auf die interne Heterogenität der sie interessierenden Gebilde. Anregungen für ein methodologisch kosmopolitisches Forschungsprogramm der Soziologie bieten sie v.a. dadurch, dass staatliche und nicht-staatliche Kontexte jenseits des Nationalstaats denkbar und in empirischer Forschung konkretisiert werden. Die Herausforderung für den Kern soziologischer Forschung besteht dann darin, makrosoziale Kontexte ,jenseits“ des Nationalstaats in Mehrebenenanalysen einzubeziehen bzw. besser noch Fälle in multiplen Kontexten zu situieren, deren Grenzen und Geltungsbereiche unklar sind.

Für soziologische Vergleiche ,jenseits“ des Nationalstaats sind drei Umstellungen erforderlich. Erstens sollte nicht allein der Nationalstaat als expliziter und impliziter Rahmen für Vergleiche in Betracht kommen. Empfehlenswert ist die zusätzliche Verwendung alternativer Vergleichskontexte, wobei darauf zu achten wäre, dass sich die gewählten Vergleichsrahmen nicht nur im geographischen Maßstab, sondern auch konzeptionell vom Staats-Zentrismus (vgl. Brenner 1999) unterscheiden. Es genügt nicht, die Europäische Union oder den ,islamischen Kulturkreis“" als Rahmen des Vergleichs zu wählen, wenn man dabei weiterhin unterstellt, dass diese Kontexte durch eindeutige territoriale Grenzen, geteilte Normen und / oder zentrale Steuerungsinstanzen gekennzeichnet sind. Auch sind große Vergleichskontexte nicht nach Art russischer Puppen ineinander verschachtelt, sondern sie werden durch quer zu nationalen Grenzen verlaufende Ströme und Verflechtungen gekreuzt (Urry 2000; Pries 2008 a). Eine kosmopolitische Perspektive auf Vergleiche ,jenseits“ des Nationalstaats sollte sich für den Gedanken öffnen, dass große Kontexte unabgegrenzt, in sich heterogen und miteinander verflochten sind. Auch können große Kontexte durchaus nicht nur territorial abgegrenzt werden, sondern auch soziale Rahmen wie z.B. inter-, supra- und transnationale (Nicht-) Regierungsorganisationen, Professionen, Märkte, Felder oder Funktionssysteme kommen als Kontexte für multiple Vergleiche in Betracht (Weiß im Erscheinen).

Das setzt zweitens inhaltliche Konzepte voraus, auf deren Grundlage Fälle in verschiedenen Kontexten platziert werden können. Wenn man sich z.B. für die Individualisierung von Lebensläufen interessiert, wäre zu fragen, ob bestimmte Formen der Wohlfahrtsstaatlichkeit eine zwingende Voraussetzung dafür sind, dass individualisierte Lebensläufe entstehen, oder ob eine Herauslösung von Menschen aus angestammten Bindungen, die diese in ein direktes Verhältnis zum Staat bringt, auch unabhängig von entwickelter Wohlfahrtsstaatlichkeit, im Verhältnis zu mehreren Staaten und zu anderen Formen entwickelter Staatlichkeit denkbar wäre. Die Definition des interessierenden Gegenstands sollte also möglichst auf unreflektierte Referenzen zu Nationalstaaten und Ethnizitäten verzichten.

Wenn man sich für die Individualisierung von Lebensläufen in der (reflexiven) Moderne interessiert, würde man folgendermaßen vorgehen: Zunächst wäre das interessierende Phänomen so zu definieren, dass es nicht explizit oder implizit auf einen spezifischen Kontext re- 
duziert wird. Man kann Individualisierung z.B. als einen Prozess ansehen, in dessen Verlauf sich direkte und plurale Verbindungen zwischen Individuen und Staatlichkeit entwickeln, die von den Individuen als individuell gestaltbar begriffen werden (vgl. Wohlrab-Sahr 1997). Dabei wird offen gehalten, ob und inwiefern ein starker nationaler Wohlfahrtsstaat Voraussetzung von Individualisierung ist und ob das Konzept des Lebenslaufs geeignet ist, um Individualisierungsprozesse weltweit zu erfassen. Dies muss empirisch untersucht werden, was direkt zur dritten Anforderung an eine kosmopolitische Analyse von Individualisierungsprozessen überleitet: Die Bedeutung von bestimmten Formen der Staatlichkeit wird erst erkennbar, wenn man in verschiedenen Kontexten nach der Herausbildung von direkten Relationen zwischen Personen und Staatlichkeit sucht und die Ergebnisse aus verschiedenen Kontexten vergleicht. Im Folgenden werden einige Möglichkeiten für Vergleiche ,jenseits“ des Nationalstaats dargestellt und anhand von Forschungsdesigns der Autorin illustriert.

\section{Methodologischer Kosmopolitismus in soziologischen Forschungsdesigns}

Empirische Forschung zu Migration ist ein geeignetes Beispiel, um die Möglichkeiten eines methodologischen Kosmopolitismus zu diskutieren, weil Migranten in mehreren nationalen und teils auch inter-, supra- und transnationalen Kontexten situiert sind. Beispielhaft soll hier auf die modernisierungstheoretische Frage eingegangen werden, wie sich Individualisierungsprozesse bei hoch qualifizierten MigrantInnen beobachten ließen. Ähnlich wie der Lebenslauf wird auch das an ihn geknüpfte Konzept der Individualisierung durch Staatlichkeit mit strukturiert, ohne dass es vollständig und eindeutig auf bestimmte staatliche Kontexte zugerechnet werden könnte. ${ }^{6}$

Wie gezeigt, sollte der interessierende Gegenstand zunächst so definiert werden, dass er für unterschiedliche Vergleichskontexte geeignet ist. Für eine methodologisch kosmopolitische Perspektive auf Individualisierungsprozesse in der Migration würde das einerseits bedeuten, dass man auch für Migranten und Migrantinnen unterstellt, dass diese einen individuellen Lebenslauf entwickeln können. Das klingt banal, ist aber insofern nicht selbstverständlich, als ein großer Teil der Migrationsforschung Migranten als Kollektiv definiert und untersucht (zur Kritik vgl. Wimmer/Glick Schiller 2002). Auch das in der Forschung überwiegend verwendete Muster des institutionalisierten Lebenslaufs bedarf einer Anpassung. Die Lebensläufe von Migranten und Migrantinnen beinhalten nicht nur Statusübergänge zwischen Bildungswesen und Arbeitsmarkt, sondern auch Statuspassagen im Staatensystem (Nohl et al. 2006). Schittenhelm spricht daher von einer mehrdimensional strukturierten Statuspassage in den Arbeitsmarkt (2005). Wenn man Lebensläufe so versteht, kann man untersuchen, inwiefern die Lebensläufe und Selbstdefinitionen von Migranten eher durch den unmittelbaren Zugriff von Staatlichkeit auf Individuen oder eher durch eine anhaltende Bedeutung von intermediären Instanzen wie Familie oder ethnischer Gruppe geprägt sind.

Allerdings können solche Fragen nicht im Rahmen eines nationalstaatlichen Kontexts beantwortet werden. Man muss die Position von Migranten im Verhältnis zu mehreren Natio-

6 Die von Beck 1986 vorgeschlagene Lesart von Individualisierung hebt hervor, dass Individualisierung einerseits als Freisetzung des Individuums aus traditionalen Kollektiven und damit als Verlust traditionaler Sicherheiten verstanden werden sollte, andererseits aber auch als ,neue Art der sozialen Einbindung" (Beck 1986: 206), die Individuen unmittelbar dem Zugriff des Staates oder auch der Erfahrung von Massenarbeitslosigkeit aussetzt. Wie Beck später selbstkritisch anmerkt, blieb die damalige Debatte methodologisch nationalistisch (Beck 2007). Heute würde er institutionalisierte Individualisierung als makrosozialen Prozess begreifen, in Verlauf dessen (Grund-)Rechte dem Individuum und nicht länger dem Kollektiv zugestanden werden. In diesem Argument wird die Bedeutung des Wohlfahrtsstaates für Individualisierungsprozesse besonders unterstrichen. In beiden Lesarten sind Individualisierungsprozesse auf widersprüchliche Weise mit Staatlichkeit verbunden, weil sie vom Staat angestoßen und ihre riskanten Folgen vom Staat aufgefangen werden. 
nalstaaten, zum Staatensystem insgesamt, zu Staatlichkeit jenseits des Nationalstaats und zu nicht- oder trans-staatlichen Kontexten analysieren, wenn man Individualisierungsprozesse in der Migration untersuchen will. Im zweiten Schritt gilt es also, Vergleichshorizonte zu definieren, in denen die strukturierende Wirkung von verschiedenen Kontexten auf Lebensläufe insgesamt erkennbar wird. Wenn man die Variation von Kontexten über das Design der Forschung abstrakt variieren will, kommen unterschiedliche Optionen in Betracht, die anhand von Forschungsprojekten der Autorin illustriert werden. Diese Forschungsprojekte arbeiten mit qualitativen Methoden, können aufgrund ihrer quasi-experimentellen Logik aber auf Forschungsdesigns übertragen werden, die prüfstatistisch generalisieren.

\subsection{Kontexte ,jenseits“ des Nationalstaats}

Einerseits ist es möglich, die Fälle gezielt in Kontexten zu platzieren, die von nationalstaatlichen Besonderheiten absehen. Im Projekt „Hochqualifizierte Migrant / innen. Zur Transnationalisierung sozialer Lagen“(Weiß 2005; 2006 a,b) wurden nur Migranten und Migrantinnen untersucht, die über ein so hohes und spezifisches Ausmaß an kulturellem Kapital verfügen, dass sie auch nach der Migration unmittelbar Zugang zu hochqualifizierten Arbeitsmärkten finden. Primärer Kontext der Analyse sind damit transnational strukturierte Arbeitsmärkte. Außerdem folgte die Auswahl des Samples einer quasi-experimentellen Logik (Weiß 2006 a), die Vergleiche zwischen Migranten, die aus Entwicklungs- und Schwellenländern nach Deutschland gewandert waren (kurz: IT-Fachkräfte), und solchen, die aus Deutschland in Entwicklungs- und Schwellenländer gegangen sind (kurz: Expatriates), ermöglichte. Sie hatten damit u.a. EU-Grenzen passiert.

Das Projekt zielte nicht auf eine Analyse von Individualisierungsprozessen ab, sondern fragte nach der Struktur transnationaler sozialer Lagen. Hinsichtlich der meisten Indikatoren für soziale Lagen zeigte sich die erwartete Abkoppelung dieser Hochqualifizierten, deren kulturelles Kapital weltweit anerkannt wird, vom nationalen Rahmen. Entsprechend war die soziale Lage der IT-Fachkräfte und der Expatriates in vielen Dimensionen vergleichbar. Ein Ergebnis steht jedoch in klarem Zusammenhang damit, ob ein Migrant in die EU oder aus der EU heraus wandert und es ist für eine kosmopolitische Perspektive auf Individualisierungsprozesse interessant: Die soziale Absicherung der IT-Fachkräfte unterscheidet sich deutlich von der der Expatriates (vgl. Weiß 2006b). Die IT-Fachkräfte mussten zu den staatlichen Sicherungssystemen Deutschlands beitragen, obwohl sie zum Zeitpunkt der Erhebung aufgrund der Ausgestaltung ihrer Visa nicht von Arbeitslosen- oder Pflegeversicherung profitieren konnten. Meist unterhielten sie deshalb auf eigene Kosten zusätzlich private Versicherungen. Einige finanzierten Verwandten oder Nachbarn im Herkunftsland eine höhere Bildung, was man als weitere Form sozialer Absicherung ansehen kann. Auch die Expatriates unterhielten zusätzlich zur Pflichtmitgliedschaft in den Sozialversicherungen private Versicherungen, die jedoch von ihren Arbeitgebern bezahlt wurden. Die Expatriates profitierten außerdem davon, dass sie ihre Einkünfte nur teilweise versteuern mussten. Laxe Regelungen der Zielländer, ungenügende Kontrollen und eine gängige Praxis, Einkünfte an der Steuer vorbeizuschleusen, führten dazu, dass die Expatriates bei minimalen eigenen Beiträgen durch staatliche und private Sicherungssysteme und durch die Möglichkeit, als Staatsbürger in entwickelte Wohlfahrtsstaatlichkeit zurückzukehren, dreifach sozial abgesichert waren, während sich die IT-Fachkräfte mehrfach absichern mussten, weil sie zu Pflichtbeiträgen ohne erwartbare Leistungen gezwungen sind. Die Institutionen des Wohlfahrtsstaats sind nicht darauf ausgerichtet, dass Menschen ein- und womöglich nach kurzer Zeit weiterwandern. Wenn eigene Staatsbürger beruflich im Ausland tätig sind, bleiben sie durch eine Reihe von Mitgliedschaften Bürger eines starken Wohlfahrtsstaats. Für Staatsangehörige von Entwicklungs- und Schwellenländern wirkt sich die starke Wohlfahrtsstaatlichkeit der Zentren hingegen negativ aus, solange sie nicht definitiv einwandern und zu Staatsbürgern werden. Auch bei ihnen kann aber von 
einem individualisierten Zugriff der Wohlfahrtsstaatlichkeit auf ihre Lebenschancen gesprochen werden.

Die strukturierende Wirkung eines Systems ungleich mächtiger Staaten für soziale Lagen lässt sich nur in einem Forschungsdesign erkennen, das das Verhältnis von Migranten zu mehreren Staaten und deren Zusammenschlüssen zum Gegenstand von Vergleichen macht. Allerdings sieht dieses Forschungsdesign so sehr von Besonderheiten einzelner Staaten ab, dass Ergebnisse, die das Verhältnis von Migranten zu spezifischen Nationalstaaten klären, die Ausnahme bleiben.

\subsection{Theoretisch definierte Kontrasthorizonte für einen nationalen Kontext}

Alternativ hierzu kann man einen nationalstaatlich definierten Kontext ins Zentrum stellen, und die Partikularität dieses Kontextes durch gezielte Vergleiche erhellen. Die internationale Studiengruppe „Kulturelles Kapital in der Migration. Zur Bedeutung von Bildungs- und Aufenthaltstiteln während der Statuspassage in den Arbeitsmarkt", die die Autorin zusammen mit A.-M. Nohl, K. Schittenhelm und O. Schmidtke leitete (Nohl et al. 2006; 2010), fokussierte den deutschen Fall für eine Analyse hochqualifizierter Migranten und Migrantinnen. Im Unterschied zum Vorprojekt wurden auch Personen untersucht, die im Übergang zwischen Nationalstaaten Nachteile erleiden, sei es, weil ihr ausländischer Bildungstitel nicht anerkannt wird, oder weil sie über einen im Vergleich zu Einheimischen nachrangigen Rechtsstatus verfügen. Die untersuchten Fallgruppen wurden theoretisch begründet z.B. als rechtlich nachrangige Bildungsausländer definiert und nicht über nationale Zugehörigkeit.

In diesem Projekt war es möglich, die strukturierende Wirkung des deutschen Kontextes empirisch zu untersuchen, weil die theoretisch definierten Statusgruppen jeweils auch in einem zu Deutschland stark kontrastierenden nationalen Kontext untersucht wurden. Durch die Analyse mehrerer Statusgruppen in insgesamt vier Ländern wurde außerdem erkennbar, wenn spezifische nationale Kontexte keine oder nur eine geringe Bedeutung für die Statuspassage in den Arbeitsmarkt hatten. Die besonderen Bedingungen, die den im Vorprojekt untersuchten IT-Fachkräften eine freie Zirkulation ermöglichen, werden im Vergleich zu anderen Typen erkennbar, deren Statuspassage in den Arbeitsmarkt sehr viel stärker durch nationalstaatliche und EU-Instanzen strukturiert ist. In diesem Design lässt sich zeigen, ob Staatlichkeit als solche, ob suprastaatliche Regelungen oder ob Besonderheiten des deutschen Nationalstaates strukturierend wirken. Das Design bleibt aber methodologisch nationalistisch, weil es Fallgruppen stark über die Zugehörigkeit zu einem Nationalstaat definiert.

\subsection{Erproben verschiedener Vergleichskontexte im Verlauf der empirischen Analyse}

Im Verlauf der Auswertungsarbeiten im Projekt „Kulturelles Kapital in der Migration“ entwickelten Schittenhelm und Nohl eine Form des Vergleichens, die der ethnographischen und historisch vergleichenden Methode (Mahoney 2003) insofern nahesteht, als im Verlauf der Analysen verschiedene Kontexte auf ihre empirische Nützlichkeit hin geprüft werden. Nohl hat diese Vorgehensweise als „Vergleich typologisch situierter Fallgruppen“ (Nohl 2009) bezeichnet. Er schlägt vor, zunächst Typologien zu erstellen, die die Varianz der Ergebnisse innerhalb eines spezifischen nationalen Kontextes abbilden. Wenn man Typologien zu ähnlichen Themen in verschiedenen nationalen Kontexten erstellt, können dann ,typologisch situierte Fallgruppen“ über Ländergrenzen hinweg verglichen werden.

Ein Erproben verschiedener Vergleichskontexte im Verlauf der Analyse ermöglicht die empirisch begründete Feststellung, dass bestimmte Phänomene nicht im Nationalstaat, sondern z.B. in transnational strukturierten Märkten und Professionen kontextualisiert werden müssen. 
Das betrifft bei dem hier vorgestellten Forschungsprojekt z.B. die Fallgruppe der Manager (Nohl 2010: 158ff), die in allen untersuchten nationalstaatlichen Kontexten zunächst die „lokalen Codes des Arbeitens", insbesondere die verhandlungssicheren Sprachkenntnisse und Feinheiten der Interaktion, erlernen müssen. Analysen dieser Fallgruppe in verschiedenen nationalen Kontexten zeigen, dass lokale Besonderheiten die transnational strukturierte Profession der Manager nur am Rande prägen, d.h. auch wenn die Manager in Deutschland eher als Trainees einsteigen und in Kanada eher im Zuge niedrig qualifizierter Beschäftigungsverhältnisse, wäre es verfehlt, die Statuspassage in den Arbeitsmarkt als „,deutsch“ oder ,kanadisch“ zu begreifen (vgl. Nohl 2010: 158ff).

Hingegen kommt ein Vergleich von für Deutschland und die Türkei erstellten Typologien rechtlicher Exklusion zu dem erwartbaren Befund, dass rechtliche Exklusion stark durch nationalstaatliche Kontexte und supra- und internationale Regelungen (Hollifield 2000) bestimmt wird. Allerdings sind nur wenige Typen rechtlicher Exklusion durch historische Besonderheiten eines bestimmten Nationalstaats erklärbar (Weiß / Ofner / Pusch 2010). Eine solche Ausnahme ist z.B. die rechtliche Exklusion von Asylbewerbern, die in Deutschland zwar vollständig vom Arbeitsmarkt ferngehalten werden, aber eine minimale Absicherung erhalten. Im Unterschied dazu ist für die Asylbewerber in der Türkei das UNHCR zuständig, das die Flüchtlinge nicht versorgen kann, so dass sie trotz Arbeitsverbot informell arbeiten und das aufgrund seltener Kontrollen auch können. Überwiegend zeigt sich durch die Vergleiche zu rechtlicher Exklusion, dass spezifische Nationalstaaten aufgrund von international geteilten Standards nur geringe Handlungsspielräume für eine eigenständige Politikgestaltung haben. So ist es für Nationalstaaten offenbar kaum möglich, die Ehepartner von Staatsbürgern bleibend vom Arbeitsmarkt auszuschließen. Trotz deutlich verschiedener rechtlicher Regelungen in Deutschland und der Türkei haben hochqualifizierte Ehepartner von Staatsbürgern einen vergleichbar guten Arbeitsmarktzugang.

Auch die Studie „Kulturelles Kapital in der Migration“ untersuchte nicht primär Individualisierungsprozesse. Wenn man sich für die Frage nach Individualisierungsprozessen in der Migration interessiert, fällt aber auf, dass sich ein deutlich entindividualisierender Einfluss des Ausländerrechts auf die Lebensführung nachweisen lässt: Soweit die EU und spezifische Staaten in der EU Einwanderung unterbinden wollen, stellt die Familienzusammenführung oft die einzige Möglichkeit einer legalen Zuwanderung dar. Die von uns befragten Migranten und Migrantinnen thematisierten entsprechend oft Widersprüche zwischen ihrem Anspruch auf individuelle Lebensführung und Strukturen, in denen sie zur Heirat und zu einer Selbstdefinition als Partner einer oder Mutter eines Deutschen genötigt werden.

Ein Erproben verschiedener Vergleichskontexte im Verlauf der empirischen Analyse ermöglicht empirisch fundierte Unterscheidungen zwischen Phänomenen, die nicht durch Nationalstaaten strukturiert sind (z.B. das professionelle Feld der Manager), und solchen, die nur im Kontext eines oder mehrerer Nationalstaaten verständlich sind. Außerdem kann man untersuchen, wie das Staatensystem insgesamt bzw. inter- und supranationale staatliche Regime auf Lebensläufe wirken. Dass Ausländer rechtliche Nachteile erleiden, ist z.B. kaum einzelnen Staaten anzurechnen, sondern geht darauf zurück, dass die territoriale Segmentierung des Funktionssystems Politik Ungleichheitsschwellen an den Grenzen nationaler Wohlfahrtsstaaten errichtet (Bommes 1999). Die Lebensverläufe von Flüchtlingen, die nach Deutschland gelangen, werden hingegen sehr deutlich durch pfadabhängige Entwicklungen in Deutschland strukturiert.

\section{Schluss}

In Zeiten der Globalisierung ist die eingangs zitierte Gegenüberstellung von nomothetischen und ideographischen Disziplinen überholt. Ehemals ideographische Disziplinen wie die ,,social 
anthropology“ arbeiten am Programm einer ,global ethnography“, das makrosozialen Strukturen gerecht werden kann. Ehemals nomothetische Disziplinen wie die Soziologie werden für ihren methodologischen Nationalismus kritisiert und entwickeln alternative Paradigmen wie das eines sozialwissenschaftlichen Kosmopolitismus.

Die Vervielfältigung sozialer Kontexte fordert die Soziologie allerdings in anderer Weise heraus als die ideographischen Traditionslinien, die sich in Forschung und Theorie durchgängig mit der Spezifik der von ihr untersuchten Fälle beschäftigt haben. Das gilt insbesondere für den Kern soziologischer Forschung, der durch systematische Vergleiche Aussagen mit Anspruch auf Repräsentativität oder Generalisierbarkeit empirisch begründen will. Dieser Strang der soziologischen Forschung verzichtet häufig auf eine Reflexion des Rahmens, in dem Vergleiche situiert sind.

Das nomothetische Erbe der Soziologie könnte dann zu einer Stärke werden, wenn es zu einer systematischen Variation von Vergleichskontexten weiterentwickelt würde. Denn die soziologische Forschung kann Fälle probeweise auch in sehr abstrakten Kontexten situieren. Statt diese Kontexte vorschnell mit dem Nationalstaat zu identifizieren, sollte die soziologische Forschung Vergleiche in einer Mehrzahl von Kontexten situieren, die sich nicht nur hinsichtlich ihrer geographischen Reichweite, sondern auch hinsichtlich ihres sozialen Gehalts vom Nationalstaat unterscheiden. Die abstrakte Platzierung von Fällen in mehreren Vergleichskontexten sollte im zweiten Schritt in systematische Vergleiche von Vergleichskontexten münden, anhand derer empirisch geprüft werden kann, welche Kontexte den Fall am besten rahmen.

Man kann sich natürlich fragen, ob Rettungsversuche für eine nomothetische Tradition in der Sozialforschung überhaupt zu rechtfertigen sind. Manche Nachbardisziplinen und größere Teile der theoretischen Soziologie und der Forschung im interpretativen Paradigma würden dies bezweifeln. Einer derart grundsätzlichen Kritik kann und muss entgegengehalten werden, dass gerade Versuche einer innovativen Reformulierung der Soziologie dringend einer empirischen Forschung bedürfen, die die theoretischen Entwicklungen der letzten Jahre umsetzt und die den in der Soziologie so wichtigen Anspruch auf Allgemeingültigkeit modifiziert, ohne ihn aufzugeben. Es wird noch einiges brauchen, bis man dieses Ziel als erreicht ansehen kann.

\section{Literatur}

Albrow, Martin (1996): The Global Age, Cambridge.

Albrow, Martin (1997): Auf Reisen jenseits der Heimat. Soziale Landschaften in einer globalen Stadt, in: Ulrich Beck (Hg.), Kinder der Freiheit, Frankfurt / Main, S. 288-314.

Beck, Ulrich (1986): Risikogesellschaft. Auf dem Weg in eine andere Moderne, Frankfurt / Main.

Beck, Ulrich (2007): Beyond class and nation: reframing social inequalities in a globalizing world, in: British Journal of Sociology 58 / 4, S. 679-706.

Beck, Ulrich / Edgar Grande (2004): Das kosmopolitische Europa. Gesellschaft und Politik in der Zweiten Moderne, Frankfurt / Main.

Beisheim, Marianne / Gunnar Folke Schuppert (Hg.) (2007): Staatszerfall und Governance, Baden-Baden. Berger, Peter A. / Anja Weiß (Hg.) (2008): Transnationalisierung Sozialer Ungleichheit, Wiesbaden.

Blossfeld, Hans-Peter / Sandra Buchholz / Dirk Hofäcker / Heather Hofmeister / Karin Kurz / Melinda Mills (2008): Globalization and changes in social inequality in modern societies, in: Kölner Zeitschrift für Soziologie und Sozialpsychologie 59 / 4, S. $667-691$.

Boatcă, Manuela (2009): Class vs. Other as Analytic Categories. The Selective Incorporation of Migrants into Theory, in: Terry Ann Jones / Eric Mielants (Hg.), Mass Migration in the World-System. Past, Present, and Future, Boulder - London, S. 38-54. 
Bommes, Michael (1999): Migration und nationaler Wohlfahrtsstaat. Ein differenzierungstheoretischer Entwurf, Opladen.

Bonß, Wolfgang / Felicitas Esser / Joachim Hohl / Helga Pelizäus-Hoffmeister / Jens Zinn (2004): Biographische Sicherheit, in: Ulrich Beck / Christoph Lau (Hg.), Entgrenzung und Entscheidung. Was ist neu an der Theorie reflexiver Modernisierung?, Frankfurt / Main, S. 211-233.

Botzem, Sebastian / Jeanette Hofmann / Sigrid Quack / Gunnar Folke Schuppert / Holger Straßheim (Hg.) (2009): Governance als Prozess. Koordinationsformen im Wandel, Baden-Baden.

Bourdieu, Pierre (1984): Homo Academicus, Frankfurt / Main.

Bourdieu, Pierre (1991): Die biographische Illusion, in: BIOS 2 / 2, S. 75-81.

Brenner, Neil (1999): Beyond state-centrism? Space, territoriality, and geographical scale in globalization studies, in: Theory and Society 28 / 1, S. 39-78.

Ebbinghaus, Bernhard (1996): Spiegelwelten. Vergleich und Mehrebenenanalyse in der Europaforschung, in: Thomas König / Elmar Rieger / Hermann Schmitt (Hg.), Das europäische Mehrebenensystem, Frankfurt / Main - New York, S. 405-428.

Eisenstadt, S. N. (2000): Die Vielfalt der Moderne, Weilerswist.

Erlinghagen, Marcel / Matthias Knuth (2010): Unemployment as an Institutional Construct? Structural Differences in Non-Employment between Selected European Countries and the United States, in: Journal of Social Policy 39 / 1, S. 71-94.

Faist, Thomas (2000): The volume and dynamics of international migration and transnational social spaces, Oxford.

Gille, Zsuzsa / Seán Ó Riain (2002): Global Ethnography, in: American Review of Sociology 28, S. 271-295.

Gilroy, Paul (1993): The Black Atlantic: Modernity and Double-Consciousness, Cambridge / MA.

Glick Schiller, Nina / Ayse Caglar / Thaddeus C. Guldbrandsen (2006): Beyond the ethnic lens: locality, globality, and born-again incorporation, in: American Ethnologist 33 / 4, S. 612-633.

Goldthorpe, John H. (1997 a): Current Issues in comparative macrosociology: a debate on methodological issues, in: Comparative Social Research 16, S. 1-26.

Goldthorpe, John H. (1997 b): Current issues in comparative macrosociology: a response to the commentaries, in: Comparative Social Research 16, S. 121-132.

Heinz, Walter R. (Hg.) (2000): Übergänge. Individualisierung, Flexibilisierung und Institutionalisierung des Lebensverlaufs (ZSE Beiheft), Weinheim / München.

Hollifield, James F. (2000): Migration and the "new" international order: The missing regime, in: B. Gosh (Hg.), Managing migration: Time for a new international regime, Oxford, S. 95-109.

Hopkins, Terence K. / Immanuel Wallerstein (1967): The comparative Study of National Societies, in: Social Science Information $5 /$ 1, S. 25-58.

Iredale, Robyn (2001): The migration of professionals: Theories and typologies, in: International Migration $39 / 5$, S. 7-26.

Kohli, Martin (1985): Die Institutionalisierung des Lebenslaufs. Historische Befunde und theoretische Argumente, in: Kölner Zeitschrift für Soziologie und Sozialpsychologie 37, 1-29.

Kohli, Martin (1994): Institutionalisierung und Individualisierung der Erwerbsbiographie, in: Ulrich Beck / Elisabeth Beck-Gernsheim (Hg.), Riskante Freiheiten. Individualisierung in modernen Gesellschaften. Frankfurt / Main, S. 219-244.

Leisering, Lutz / Roberts Walker (Hg.) (1998): The dynamics of modern society. Poverty, policy and welfare, Bristol.

Levitt, Peggy / Nina Glick Schiller (2004): Conceptualizing simultaneity: a transnational social field perspective on society, in: International Migration Review 38 / 3, S. 1002-1039. 
Mahoney, James (2003): Strategies of Causal Assessment in Comparative Historical Analysis, in: James Mahoney / Dietrich Rueschemeyer (Hg.), Comparative Historical Analysis in the Social Sciences, New York, S. 337-372.

Marcus, George E. (1995): Ethnography in/of the world system: The emergence of multi-sited ethnography, in: Annual Review of Anthropology 24, S. 95-117.

Matthes, Joachim (1992): The Operation Called 'Vergleichen', in: Joachim Matthes (Hg.), Zwischen den Kulturen? Die Sozialwissenschaften vor dem Problem des Kulturvergleichs, Göttingen, S. 75-99.

Mau, Steffen (2007): Transnationale Vergesellschaftung. Die Entgrenzung sozialer Lebenswelten. Frankfurt / Main.

Mayer, Karl Ulrich / Walter Müller (1986): The state and the structure of the life course, in: Aage B. Sørensen / Franz E. Weinert / Lonnie R. Sherrod (Hg.), Human development and the life course: multidisciplinary perspectives, Hilssdale / N.J., S. 217-245.

Milanovic, Branko (2008): Where in the world are you? Assessing the importance of circumstance and effort in a world of different mean country incomes and (almost) no migration (Policy Research Working Paper 4493): The World Bank Development Research Group Poverty Team.

Mill, John Stuart (1973 [1843]): A system of logic ratiocinative and inductive. Book 3: on induction. Toronto.

Nohl, Arnd-Michael (2007): Komparative Analyse als qualitative Forschungsstrategie, in: J. Straub / A. Weidemann / D. Weidemann (Hg.), Handbuch Interkulturelle Kommunikation und Kompetenz. Stuttgart, S. 391-403.

Nohl, Arnd-Michael (2009): Der Mehrebenenvergleich als Weg zum kontextuierten Ländervergleich. Methodologische Überlegungen anhand eines internationalen Projektes zur Migrationsforschung, in: Sabine Hornberg / Inci Dirim / Gregor Lang-Wojtasik / Paul Mecheril (Hg.), Beschreiben? Verstehen? Interpretieren. Stand und Perspektiven International und Interkulturell Vergleichender Erziehungswissenschaft in Deutschland, Wiesbaden, S. 95-110

Nohl, Arnd-Michael (2010): Von der Bildung zum kulturellen Kapital: Die Akkreditierung ausländischer Hochschulabschlüsse auf deutschen und kanadischen Arbeitsmärkten, in: Arnd-Michael Nohl / Karin Schittenhelm / Oliver Schmitdke / Anja Weiß (Hg.), Kulturelles Kapital in der Migration: Hochqualifizierte Einwanderer und Einwanderinnen auf dem Arbeitsmarkt, Wiesbaden, S. 153-165.

Nohl, Arnd-Michael / Karin Schittenhelm / Oliver Schmidtke / Anja Weiß (Hg.) (2010): Kulturelles Kapital in der Migration: Hochqualifizierte Einwanderer und Einwanderinnen auf dem Arbeitsmarkt, Wiesbaden.

Nohl, Arnd-Michael / Karin Schittenhelm / Oliver Schmitdke / Anja Weiß (2006): Cultural capital during migration - A multi-level approach for the empirical analysis of the labor market integration of highly skilled migrants, in: Forum Qualitative Sozialforschung [On-Line Journal] $7 / 3$ Art. 14, http:// www.qualitative-research.net/fqs-texte/3-06/06-3-14-e.htm.

Pries, Ludger (2008 a): Die Transnationalisierung der sozialen Welt. Sozialräume jenseits von Nationalgesellschaften, Frankfurt / Main.

Pries, Ludger (2008 b): Transnational societal spaces. Which units of analysis, reference and measurement?, in: Ludger Pries (Hg.), Rethinking Transnationalism. The Meso-link of organisations. London, S. 1-20.

Przeworski, Adam / Henry Teune (1970): The logic of comparative social inquiry, New York - London - Toronto - Sydney.

Przyborski, Aglaja / Monika Wohlrab-Sahr (2008): Qualitative Sozialforschung. Ein Arbeitsbuch, München.

Quack, Sigrid (2007): Legal professionals and transnational law-making: a case of distributed agency, in: Organization 14, S. 643-666.

Ragin, Charles C. (1987): The comparative method. Moving beyond qualitative and quantitative strategies, Berkeley - Los Angeles - London. 
Randeria, Shalini (2003): Cunning states and Unaccountable International Institutions: Legal Plurality, Social Movements and Rights of Local Communities to Common Property Resources, in: European Journal of Sociology 44 / 1, S. 1-27.

Robertson, Roland (1992): Globalization. Social Theory and Global Culture. London, Thousand Oaks New Delhi.

Scheuch, Erwin K. (1968): The cross-cultural use of sample surveys. Problems of comparability, in: Stein Rokkan (Hg.), Comparative research across cultures and nations, Paris - The Hauge, S. 176-209.

Schittenhelm, Karin (2005): Soziale Lagen im Übergang. Junge Migrantinnen und Einheimische zwischen Schule und Berufsausbildung, Wiesbaden.

Schwinn, Thomas (Hg.) (2006): Die Vielfalt und Einheit der Moderne. Kultur- und strukturvergleichende Analysen, Wiesbaden.

Teubner, Günter (1997): 'Global Bukowina': legal pluralism in the world society, in: Günter Teubner (Hg.), Global law without a state, Aldershot, S. 3-28.

Tilly, Charles (1984): Big structures, large processes, huge comparisons, New York.

United Nations Development Programme (2007): Human Development Report 2007/2008. Fighting climate change: human solidarity in a divided world, Houndmills / Basingstoke - New York.

Urry, John (2000): Sociology beyond societies. Mobilities for the twentyfirst century, London.

Verwiebe, Roland (2004): Transnationale Mobilität innerhalb Europas, Berlin.

Wallerstein, Immanuel (2004): World-Systems Analysis: An Introduction. Durham / NC.

Weiß, Anja (2005): The transnationalization of social inequality. Conceptualizing social positions on a world scale, in: Current Sociology 53 / 4, S. 707-728.

Weiß, Anja (2006 a): Comparative research on highly skilled migrants. Or: (In what way) can qualitative interviews be used in order to reconstruct a class position?, in: Forum Qualitative Sozialforschung [OnLine Journal] 7 / 3 Art. 2, http://www.qualitative-research.net/fqs-texte/3-06/06-3-2-3.htm.

Weiß, Anja (2006 b): Hoch qualifizierte MigrantInnen. Der Kern einer transnationalen Mittelklasse?, in: Florian Kreutzer / Silke Roth (Hg.), Transnationale Karrieren. Biografien, Lebensführung und Mobilität, Wiesbaden, S. 283-300.

Weiß, Anja (im Erscheinen): Ungleichheit in Zeiten der Globalisierung: Buchmanuskript.

Weiß, Anja, Ulrike Selma Ofner / Barbara Pusch (2010): Migrationsbezogene biographische Orientierungen und ihre ausländerrechtliche Institutionalisierung, in: Arnd-Michael Nohl / Karin Schittenhelm / Oliver Schmitdke / Anja Weiß (Hg.), Kulturelles Kapital in der Migration: Hochqualifizierte Einwanderer und Einwanderinnen auf dem Arbeitsmarkt, Wiesbaden, S. 197-210.

Wimmer, Andreas / Nina Glick Schiller (2002): Methodological nationalism and the study of migration, in: Archives Europeennes de Sociologie 43 / 2, S. 217-240.

Wohlrab-Sahr, Monika (1997): Individualisierung: Differenzierungsprozess und Zurechnungsmodus, in: Ulrich Beck and Peter Sopp (Hg.), Individualisierung und Integration. Neue Konfliktlinien und neuer Integrationsmodus? Opladen, S. 23-36.

Zürn, Michael / Stefan Leibfried (Hg.) (2006): Transformationen des Staates? Frankfurt / Main.

Prof. Dr. Anja Weiß Institut für Soziologie Universität Duisburg-Essen Lotharstraße 65

47057 Duisburg anja.weiss@uni-due.de 